\title{
Central nervous lesions in a kidney transplant child
}

\author{
Fernández $\mathrm{C}^{1 *}$, Lázaro ${ }^{1}$, Mártinez-Bermejo $A^{2}$, Zarauza $A^{1}$ and Espinosa $L^{1}$ \\ ${ }^{1}$ Children's Nephrology Service, University Hospital “La Paz”, Madrid, Spain \\ ${ }^{2}$ Child Neurology Service, University Hospital “La Paz", Madrid, Spain
}

\section{Case report}

A nine-year-old kidney recipient with stage 3 chronic kidney disease due to poor initial graft perfusion with focal cortical infarcts, cellular rejection, calcineurin inhibitor toxicity and repeated pyelonephritis episodes presented in the Hospital Emergency Service due to a 17-day severe headache that had become more painful and accompanied by vomiting in the last two days. There was no history of trauma, infection or vaccines in the last weeks before the symptoms began.

The patient's immunosupression regime currently included Rapamicin and methylprednisolone ( $2 \mathrm{mg}$ every other day). Being a carrier of a heterozygotic mutation in Leiden's Factor V, the patient was also receiving low molecular weight heparin (LWMH) as prophylaxis for an earlier orthopedic surgery.

The patient's primary disease was a renal dysplasia and neurogenic bladder secondary to a sacro-coccygeal teratoma extirpated on his first day of life. He developed spastic paraparesia but his intellectual development was normal.

Physical examination was normal with a Glasgow score of 15. Arterial pressure was $140 / 87 \mathrm{mmHg}$

Laboratory work showed no signs of hemorrhage or infection.

A non-contrast CT scan showed an expansive lesion in the left hemisphere with mass effect, swelling of the supratentorial ventricular system and signs of obstructive hydrocephalia. Consequently, the patient was transferred to the Pediatric Intensive Care Unit.

Non-contrast MRI was performed (Figure 1) revealing images of altered corticosubcortical intensity in both hemispheres, more marked on the left side (hypointense on T-1 sequences and hyperintense on T-2 and FLAIR weighted sequences). The expansive mass effect resulted in supratentorial ventriculomegalia.
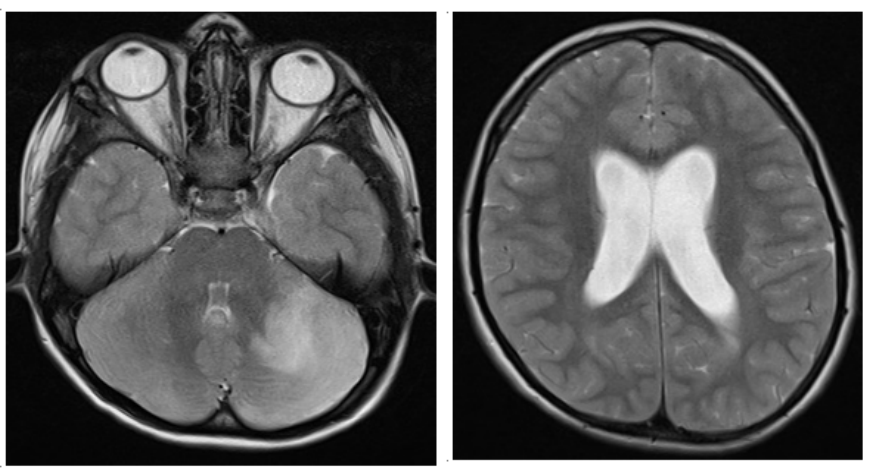

Figure 1. Initial MRI without contrast: expansive lesion in left hemisphere with mass effect and signs of obstructive hydrocephalia

An external ventricular stent was place and a sample of cerebrospinal fluid (CSF) taken; the resulting cytochemistry was normal. Microbiology studies were negative for bacteria, fungi, Herpes simplex I and II, Varicella- Zoster virus, Cytomegalovirus, Epstein Barr, Herpes 6, Enterovirus, Paraechovirus, JC virus and Cryptococcus.

Dexamethason, vancomycin and acyclovir were administered.

The patient's clinical symptoms improved within 48 hours of commencing treatment.

The non-contrast MRI on day six after admission continued to show lesions in the cerebellum despite the decreased edema and ventriculomegalia. Cerebellar biopsy was done.

The histological study reported focal demyelinization with chronic meningeal inflammation and abundant macrophages without tumoral proliferation. The immunohistochemical studies for viral involvement were negative. Cultures to detect bacteria, micobacteria and fungi were also negative. PCR studies were all negative.

Vancomycin was discontinued when the ventricular drainage was removedand Acyclovir when the biopsy results discounted viral infection. After 21 days dexamethasone was replaced with a prednisone-tapering regime.

The clinical presentation, tumor-like appearance of the lesion and other data in MR images combined with the negative microbiological results led to a preliminary differential diagnosis of demyelinizing disease vs CNS neoplasia. The histological results from the biopsy resulted in a definitive diagnosis of Schilder's Disease.

Two months later the control MRI (Figure 2) showed a marked decrease in the size of the cerebellar lesion, the disappearance of the mass effect and normal ventricular size. There was a small subacute focal lesion with hemorrhagic component in the area of the biopsy.

At present, six years after the SD episode, the patient remains asymptomatic.

\section{Discussion and conclusion}

Faced with a kidney transplant patient presenting neurological symptoms and cerebral lesions on the image studies differential diagnosis should consider infections, neoplasias, drug toxicity, cerebral vascular accidents and demyelinizing diseases.

*Correspondence to: Fernández C, Children's Nephrology Service, University Hospital "La Paz", Madrid, Spain, E-mail: carlotam.fernandez@salud.madrid.org Key words: transplant, neurological complication, demyelinizing diseases

Received: January 18, 2021; Accepted: January 26, 2021; Published: January 29, 2021 

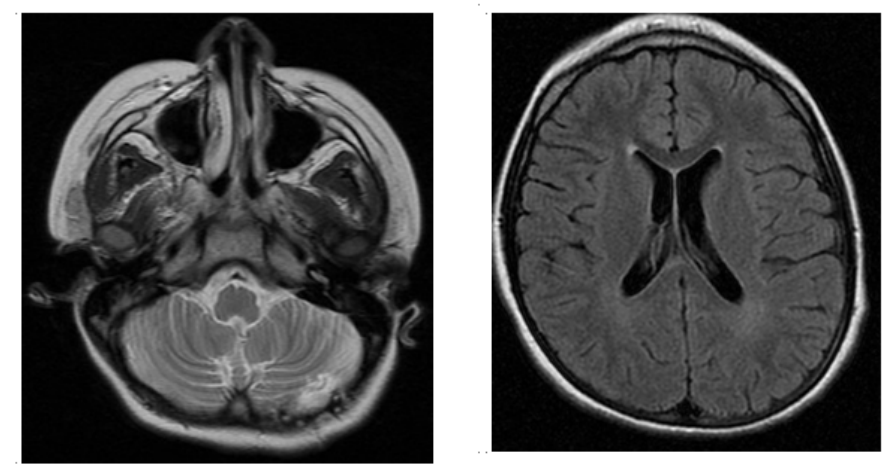

Figure 2. MRI without contrast after two months of steroid treatment: normal ventricular system. Small focal lesion with hemorrhagic component secondary to biopsy

CNS infections are the main cause of neurological complications in transplant recipients and usually occur in the first-year posttransplant [1]. Immunosuppresive therapy is the most important etiological factor. Focal CNS infectious lesions or brain abscesses have been documented in $0.36-1 \%$ of the transplant recipients with mycelial fungi being the most frequent cause. Meningoencephalitis is predominantly due to viruses, e.g., herpes viruses, and less frequently, Listeria monocytogenes, Toxoplasma gondii, and Cryptococcus [2].

The clinical presentation of infection may include symptoms such as fever, headache, altered consciousness or focal neurological deficits. When there is infection the imaging studies show hypodense or intense lesions with ring- enhancement, perilesional edema and a mass effect on adjacent structures. CSF examination may help identify the infectious agent but occasionally a cerebral biopsy is needed to make a definitive diagnosis and decide treatment.

The risk of systemic lymphoma in the first-year post kidney transplant is 20 -fold higher than in the general population. The most frequent primary cerebral neoplasia's in transplanted patients are $B$ cell lymphomas, usually associated with Epstein-Barr virus (EBV) infection and the intensity of immunosuppression. The incidence is higher in the first-year post transplant and, in EBV seronegative children under 10 years of age, tumors may occur within the first three months post-transplant $[3,4]$. Other neoplasms like glioblastomas or oligodendrogliomas are rare. Metastases (particularly of skin tumors) should also be considered.

Imaging tests (CT and MRI) are essential for diagnosis and show single or multiple masses with ring enhancement on contrast administration, but biopsy is often necessary to distinguish lymphomas from other conditions.

The prevalence of stroke among renal transplant recipients is around $8 \%$ in Spanish series [5]. Risk factors include age, diabetes, hypertension, peripheral vascular disease or prior stroke [6]. Radiological images of a cerebrovascular accident can reveal ischemic or/ and hemorrhagic infarcts.

Calcineurin-inhibitor drugs may cause symptoms, such as tremor, paresthesia, headache, seizures, optic neuropathy, hearing loss and ataxia, among others. Toxicity is imperfectly correlated with drug level and can occur within the therapeutic range, after just a few doses, or several years after initiation of therapy [4]. MRI is the best imaging test to distinguish toxicity from an infection or vascular damage. Often these lesions can be reversed by decreasing or suspending the drug [7].

If MRI suggests demyelinization, differential diagnosis should include diseases like Acute disseminated encephalomyelitis
(EMDA), Adrenoleukodystrophy (ALD), Multifocal progressive leukoencephalopathy (PML), Multiple sclerosis (MS) and Schilder's Disease (SD).

a) Acute disseminated encephalomyelitis (ADEM) is an acute autoimmune demyelinizing disease with sudden onset of multifocal neurologic deficits with encephalopathy. Most of cases are triggered by vaccination or infection. The CSF shows pleocytosis and/or mild increases in protein concentration. On magnetic resonance there are signs of newly developed diffuse, poorly demarcated, large bilateral lesions predominantly involving the cerebral white matter, although deep gray matter lesions may be present. Diagnosis is based on the clinical and radiological features [8].

b) Adrenoleukodystrophy (ADL) is an X-linked disorder characterized by progressive central demyelinization asssociated with adrenal cortex insufficiency due to an abnormal accumulation of saturated very long chain fatty acids (VLCFA) in these tissues secondary to defective peroxysomal $B$-oxidation. The different phenotypes of the disease are classified depending on the age at symptom onset [9]. Family history, clinical findings and increase of VLCFA in males with compatible symptoms, suggest the presence of this disease.

c) Progressive multifocal leukoencephalopathy (PML) is a severe demyelinating disease secondary to an opportunistic infection of JC virus in CNS white matter in immunosupressed patients. Clinical symptoms are insidious including subacute neurologic deficits, altered mental status, and visual symptoms [10]. On MRI the lesions of PML are seen as areas of decreased signal on T1-weighted images and increased signal on T2-weighted and FLAIR with preferential location in the subcortical white matter [11]. The gold standard for diagnosis of PML is brain biopsy, but it can alternatively be established by PCR detection of JC virus DNA in CSF.

d) Multiple sclerosis (MS) is an immune-mediated inflammatory demyelinating disease, rare in children, characterized by repeated CNS demyelinization episodes that are separated in time (two or more episodes of neurological deficits separated by at least a month) and location (one or more independent lesions within the CNS). Clinical features will depend on the area of the brain, optic nerves, or spinal cord affected by the acute inflammation and demyelination. In children isolated optic neuritis, isolated brain-stem dysfunction or symptoms of encephalopathy are the most common forms of presentation [12]. In adults, an elevated immunoglobulin G (IgG) index or the presence of CSF-restricted IgG oligoclonal bands (OCB) are biological hallmarks of MS, but earlier-onset pediatric MS patients may have a different CSF profile and the percentage of pediatric MS patients with OCB (8\%-92\%), an elevated IgG index (64\%-75\%), or pleocytosis $(33 \%-73 \%)$ varies widely between studies [13]. A typical MRI pattern is that of multiple well-demarcated lesions in the periventricular, juxtacortical, infratentorial, and spinal cord white matter. These areas of demyelination are best recognized on T2-weighted sequences. Enhancement of areas with active inflammation and a affected blood-brain barrier can be seen on T1 gadolinium contrast sequences. At onset in pediatric MS, T2 shows more bright lesions in the posterior fossa and more gadolinium enhancing lesions than in adults [14] The diagnosis is based on the demonstration of disseminated CNS lesions in both space and time based on clinical findings alone or combined with MRI findings [8].

e) Schilder's disease, also known as myelinoclastic diffuse sclerosis, is an infrequent subacute or chronic demyelinizing process with unknown etiology that has been considered a rare pediatric variant of MS. It presents clinically as a single or more pseudotumoral 
lesions and poses differential diagnosis with a tumor or an abscess [15]. The diagnostic criteria were published by Pose, et al. in 1986 and are still controversial. The definitive diagnosis of SD remains brain biopsy showing demyelinized areas with inflammatory infiltration (lymphocytes and macrophages) and microglial proliferation [16]. Characteristic MRI findings include uni or bilateral plaque-like lesions that appear hypodense on $\mathrm{T} 1$ sequence and hyperintense on T2 weighted images. A mass effect is common, but not always present. Contrast enhancement of the lesion margins can occur during the acute inflammatory stage. The most frequent site is the centrum semiovale, but lesions may be encountered in other locations. Advances in neuro-imaging have made it possible to diagnose this disease and rule out brain tumors or abscess without a biopsy in most cases $[17,18]$. Steroid treatment usually provides fast radiological and clinical improvement despite the frequent sequelae and possible relapses.

\section{References}

1. Ponticelli C, Campise MR (2005) Neurological complications in kidney transplant recipients. J Nephrol 18: 521-528. [Crossref]

2. Singh N, Husain $S$ (2000) Infections of the central nervous system in transplant recipients. Transpl Infect Dis 2: 101-111. [Crossref]

3. Opelz G, Döhler B (2004) Lymphomas after solid organ transplantation: a collaborative transplant study report. Am J Transplant 4: 222-230. [Crossref]

4. Pruitt AA, Graus F, Rosenfeld MR (2013) Neurological complications of solid organ transplantation. Neurohospitalist 3: 152-166. [Crossref]

5. Oliveras A, Roquer J, Puig JM, Rodriguez A, Mir M, et al. (2003) Stroke in renal transplant recipients: epidemiology, predictive risk factors and outcome. Clin Transplant 17: 1-8. [Crossref]

6. Findlay MD, Thomson PC, MacIsaac R, Jardine AG, Patel RK, et al. (2016) Risk factors and outcome of stroke in renal transplant recipients. Clin Transplant 30: 918924. [Crossref]
7. Bechstein WO (2000) Neurotoxicity of calcineurin inhibitors: impact and clinical management. Transplant Int 13: 313-326. [Crossref]

8. Krupp LB, Tardieu M, Amato MP, Banwell B, Chitnis T, et al. (2013) International Pediatric Multiple Sclerosis Study Group criteria for pediatric multiple sclerosis and immune-mediated central nervous system demyelinating disorders: revisions to the 2007 definitions. Mult Scler 19: 1261-1267. [Crossref]

9. Gärtner J, Braun A, Holzinger A, Roerig P, Lenard HG, et al. (1998) Clinical and genetic aspects of X-linked adrenoleukodystrophy. Neuropediatrics 29: 3-13. [Crossref]

10. Tan CS, Koralnik IJ (2010) Beyond progressive multifocal leukoencephalopathy: expanded pathogenesis of JC virus infection in the central nervous system. Lancet Neurol 9: 425-437. [Crossref]

11. Sahraian MA, Radue EW, Eshaghi A, Besliu S, Minagar A (2012) Progressive multifocal leukoencephalopathy: a review of the neuroimaging features and differential diagnosis. Eur J Neurol 19: 1060-1069. [Crossref]

12. Renoux C, Vukusic S, Mikaeloff Y, Edan G, Clanet M, et al (2007) Natural history of multiple sclerosis with childhood onset. $N$ Engl J Med 356: 2603-2613. [Crossref]

13. Chabas D, Ness J, Belman A, Yeh EA, Kuntz N, et al. (2010) Younger children with MS have a distinct CSF inflammatory profile at disease onset. Neurology 74: 399-405. [Crossref]

14. Verhey LH, Branson HM, Shroff MM, Callen DJ, Sled JG, et al. (2011) MRI parameters for prediction of multiple sclerosis diagnosis in children with acute CNS demyelination: a prospective national cohort study. Lancet Neurol 10: 1065-1073. [Crossref]

15. Garrido C, Levy- Gomes A, Teixeira J, Temudo T (2004) Schilder's disease: two new cases. Rev Neurol 39: 734-738. [Crossref]

16. Poser CM, Goutière F, Carpentier MA, Aicardi J (1986) Schilder myelinoclastic diffuse sclerosis. Pediatrics 77: 107-112. [Crossref]

17. Yilmaz Y, Kocaman C, Karabagli H, Ozek M (2008) Is the brain biopsy obligatory or not for the diagnosis of Schilder's disease? Review of the literature. Childs Nerv Syst 24: 3-6. [Crossref]

18. Bacigaluppi S, Polonara G, Zavanone ML, Campanella R, Branca V, et al. (2009) Schilder's Disease: non-invasive diagnosis? Neurol Sci 30: 421-430. [Crossref]

Copyright: ( 02021 Fernández C. This is an open-access article distributed under the terms of the Creative Commons Attribution License, which permits unrestricted use, distribution, and reproduction in any medium, provided the original author and source are credited. 